\title{
Quantum chemical study of the thiiranium ion intermediates and regioselectivity features of the halogenide addition
}

\author{
Ausra Vektariene $^{* \mathrm{a}, \mathrm{b}}$ and Gytis Vektaris ${ }^{\mathrm{b}}$ \\ ${ }^{a}$ Institute of Biochemistry, Mokslininku 12, LT-08662 Vilnius, Lithuania \\ ${ }^{b}$ Institute of Theoretical Physics and Astronomy of Vilnius University, A. Gostauto 12, LT-01108 \\ Vilnius, Lithuania \\ E-mail: avekt@bchi.lt
}

\begin{abstract}
Electrophilic addition reactions of the methyl sulfide cation to series of substituted ethenes leading to thiiranium intermediate formation followed by further nucleophilic attack of the chloride anion have been studied by means of $a b$ initio Hartree-Fock calculations at the $6-311+\mathrm{G}$ level. The electronic states of differently substituted thiiranium intermediate structures were investigated, in order to get more insight into the nature of the experimentally observed regioselectivity of nucleophilic attack of the chloride anion on the $C(\alpha)$ or $C(\beta)$ carbon atoms with different substituents on $\alpha$-carbon of thiiranium intermediate.

The regularities obtained in these calculations are shown to correlate well with experimental data. The quantum chemical estimation for this key reaction may be useful for experimental organic chemist in their future synthetic studies to predict structures of supposed new products for this reaction.
\end{abstract}

Keywords: Ab initio calculations, thiiranium ion, addition reaction, regioselectivity

\section{Introduction}

Ring opening reactions of three-membered heterocycles play important roles in synthetic organic chemistry, in biocatalytic reactions and in a variety of biologically relevant processes. ${ }^{1}$ Important examples of the latter are thiiranium based thioalkyl halogenides that are desirable agents in biochemistry as inhibitors of proteolytic enzymes and in medicinal chemistry as anticancer agents. $^{2}$

To obtain thioalkyl halogenides synthetic chemists mainly use electrophilic addition reactions of sulfenyl halogenides to the double bonds of functionalized alkenes. ${ }^{3}$ As shown in scheme 1, the reaction mechanism involves two main steps: first - formation of a stable intermediate, second - subsequent nucleophilic displacement of halide to give the chloroalkyl 
sulfide product. It was determined experimentally that the reaction occurs in a stereospecific trans manner, with the nucleophile (halide) attacking one of the unsaturated carbon atoms. ${ }^{3,4}$

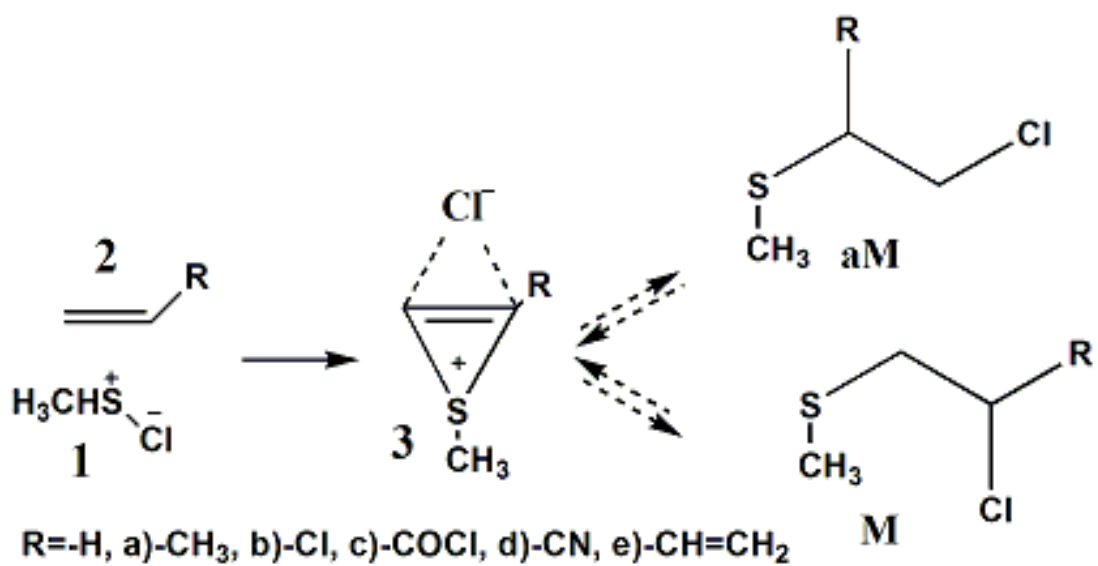

\section{Scheme 1}

The first step is usually reversible so it is the second step that determines the stereochemistry of the products. It is well known that the addition reactions of sulfenyl chlorides to unsymmetrical alkenes give two isomeric adducts, the Markownikoff adduct (M) and the antiMarkownikoff adduct (aM). The ratio of the amounts of these isomeric adducts formed is characteristically different, according to the type of the unsymmetrical alkene used. ${ }^{4}$ It was shown (scheme 1) that when the $\alpha$-carbon substituent R of intermediate structure type 3 is alkyl, chloride the predominant ring-opening reaction occurs by attack at the terminal $\beta$ carbon atom, giving the aM products. In case of methyl substituted intermediate 3a ring opening by chloride anion gives kinetically controlled aM adducts, which undergo further rearrangement to the thermodynamically stable $\mathbf{M}$. While chloro substituted intermediate $\mathbf{3 b}$ gives aM products which did not show tendencies to rearrange. ${ }^{4}$ In contrast, the addition of sulphenyl chloride to conjugated olefins when the $\alpha$-carbon substituents $\mathrm{R}$ are vinyl, carbonyl halogenide and nitrilo (intermediates 3c-e) gives $\mathbf{M}$ oriented adducts. Intermediates 3c,d substituted with carbonyl and nitrile groups gives kinetically controlled $\mathbf{M}$ adducts which undergo further rearrangement to the thermodynamically stable aM product, while in case reaction of chloride with vinyl substituted intermediate 3e obtained $\mathbf{M}$ oriented adducts are stable and did not show tendencies to rearrange.

Recent experimental studies ${ }^{1,4-7}$ of addition reactions of sulfenyl chlorides to substituted olefins have investigated the effects of the thiiranium ion intermediates and confirmed the reaction mechanism. These investigations suggest proposition that the electrophilic addition reaction mechanisms is concerned with the stable conformations of sulfonium ion intermediates: carbonium like - open classical, symmetrical bridged and unsymmetrical bridged. ${ }^{4-7}$ The ratios of the amounts of the two isomers formed are believed to result from competition between steric and electronic effects of thiiranium intermediate. ${ }^{5-7}$ Despite the extensive research that has been carried out on electrophilic addition to olefins, theoretical protocol for encompassing mechanism 
of this class reactions is still not available. Much of the discussions of the mechanisms involve reaction energetics and transition state studies ${ }^{8}$ while little effort has been expended on a molecular orbital approach to these problems. ${ }^{9}$

Addition reactions of methylsulfenyl chloride 1a-c to ethenes substituted with methyl, chloro, vinyl, carbonyl chloride, nitrilo groups 2a-d was investigated in this paper using quantum chemical Ab-initio methods in vacuum and solvated media at $6-311+\mathrm{G}$ basis set level. It was made effort to understand effects determining regioselectivity features of ring-opening reactions of thiiranium ion bearing different substitutes on $\alpha-\mathrm{C}$ using molecular orbital approach and electronic structure investigation. The quantum chemical estimation of reactivity indexes (Lowdin charges and electrophility Fukui functions) for this reaction may be useful for experimental organic chemist in their future synthetic studies to predict structures of supposed new products.

\section{Calculation procedure}

$\mathrm{Ab}$ initio Hartree-Fock calculations using $6-311+\mathrm{G}$ basis set was carried out for the addition reaction of starting reactants and intermediate structures. Full geometry optimization and other quantum chemical calculations were carried out using the GAMESS program package. ${ }^{10}$ Calculations of starting reactants were performed in vacuum and also using the polarizable continuum model (PCM) approach, to treat solution in polar $\mathrm{CH}_{2} \mathrm{Cl}_{2}$ solvent. The visualization of optimized geometries was performed with the MOLEKEL ${ }^{11 \mathrm{a}}$ and ViewMol3D ${ }^{11 \mathrm{~b}}$ programs.

\section{Results and Discussion}

The electronic states and molecular orbitals of optimized methanesulfenyl chloride in solvated media (PCM approximation for $\mathrm{CH}_{2} \mathrm{Cl}_{2}$ solvent) -1a, methanesulfenyl chloride in vacuum $\mathbf{1 b}$ was investigated and compared to those with the methanesulfenyl cation 1c (figure 1).

The Lowdin charge distribution of methanesulfenyl chloride 1a-b presented in figure 1 shows that the positive charge is mainly located on the sulfur atom, while negative charge is on the chlorine atom. The charge polarization between those atoms is enhanced by the influence of solvation effects. Moreover, the increase of positive charge density on the sulfur atom in the methylsulfide cation 1c compared to that in methylsulfenyl chloride 1a-b is so significant that becomes +0.86 . Comparison of $\mathbf{1 a}$ and $\mathbf{1 b}$ shows (figure 1) that the solvation effects reflect tendencies to lower the bond dissociation energy and bond order between the $\mathrm{S}$ and $\mathrm{Cl}$ atoms while the later for $\mathrm{C}-\mathrm{S}$ bond remains almost the same. Dissociation energy for $\mathrm{C}-\mathrm{S}$ bond is about $85 \mathrm{kcal} / \mathrm{mol}$ higher then for $\mathrm{S}-\mathrm{Cl}$ bond. These results imply that the polarization of the $\mathrm{S}-\mathrm{Cl}$ bond (up to dissociated bond level) in the methylsulfenyl chloride molecule is expected to be sensitive to the polarity of the solvent. This is important for electrophilic addition reactions, which may therefore occur fairly easily in polar solvents. Therefore we considered the methylsulfide cation 
1c for further calculations as most plausible electrophilic starting species for the initiation of electrophilic addition reaction to olefins. The distribution of the frontier molecular orbitals of the methylsulfide cation 1c was presented in the figure 2 as the most possible starting species of the addition reaction. The electrons of the highest occupied molecular orbital (HOMO) of 1a are mainly localized in the $\mathrm{p}_{\mathrm{y}}$ atomic orbital (AO) of the $\mathrm{S}$ atom and the lowest occupied molecular orbital (LUMO) is localized in the $\mathrm{p}_{z}$ of the $\mathrm{S}$ atom, which implies that the methylsulfide cation 1c can easily react with various olefins or nucleophiles forming the cationic $\pi$-type intermediates. As presented in supporting information (tables S1-S4) the calculated charge distribution and molecular orbitals of starting ethanes $\mathbf{2}$ and 2a-e and thiiranium ions $\mathbf{3}$ and 3a-e in vacuum did not show significant changes comparing to those calculated for solvated media. Further in this paper presented calculation results for solvated media using PCM method.

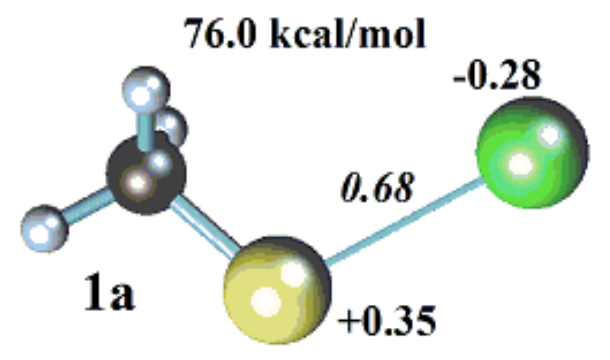

$188.0 \mathrm{kcal} / \mathrm{mol}$

$-0.17$

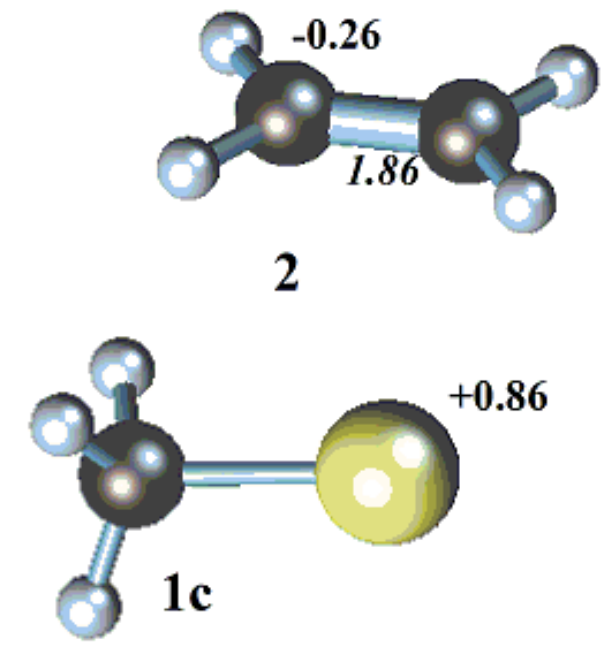

2
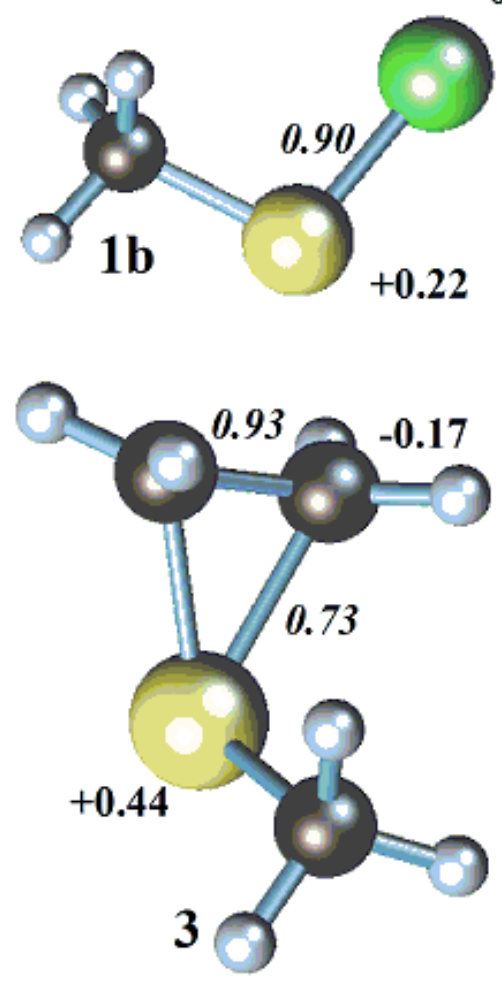

Figure 1. Lowdin charge densities, bond orders (italic) and S-Cl bond dissociation energies (presented in $\mathrm{kcal} / \mathrm{mol}$ ) of methanesulfenyl chloride calculated with PCM solvent approximation (for $\mathrm{CH}_{2} \mathrm{Cl}_{2}$ ) $\mathbf{1 a}$ and in vacuum $\mathbf{1 b}$ and methanesulfide cation $\mathbf{1 c}$, ethane 2, thiiranium ion intermediate 3 .

Secondly the study of electronic structure, molecular orbital spatial distribution and energetics of starting reactants 1c, 2 and thiiranium intermediate 3 was implemented. The 
molecular orbital diagram, presented in figure 2 and figure 1, of interacting electrophile methylsulfide cation 1c, nucleophile - ethane 2 and the reaction intermediate - thiiranium cation 3 suggest, that in the electrophilic addition the double bond transfers to the $\mathrm{S}$ atom, to neutralize almost completely the positive charge on the $\mathrm{S}$ atom and then form a fairly strong bond between the $\mathrm{S}$ and $\mathrm{C}$ atoms, resulting in weakening of the $\mathrm{C}=\mathrm{C}$ double bond. Deformation of the double bond is also caused in the process of the methylsulfide group approaching the double bond to form the $\pi$-complex thiiranium ion intermediate.

The comparison of HOMO and LUMO orbital shapes (figure 2) for the reacting ethane 2 and methylsulfide cation 1c and the thiiranium ion 3 indicate that the charge of $\pi$ electrons in the double bond transfers to the vacant orbital on the $\mathrm{S}$ atom, and otherwise the positive charge of the isolated methanesulfenyl cation wholly distributes over the intermediate. Such charge delocalization makes a contribution to the stabilization of the intermediate. Furthermore as we discuss about the process in which the nucleophile (chloride anion) attacks $\alpha$ or $\beta$ carbon atoms of cationic intermediate causing regioselective ring opening reaction. In this process, the lowest vacant orbitals of the thiiranium ion intermediates play an important role in predicting the reaction path and the stability of the final adducts.

As pictured in figure 2, the lowest vacant molecular orbitals, i.e. the LUMO and LUMO +1 of the stabilized thiiranium ion intermediate, are the Walsh-type orbitals, ${ }^{12}$ which are very close in energy. The LUMO +1 orbital is symmetrically delocalized $\pi$-type orbital on the $\mathrm{C}=\mathrm{C}$ bond and the LUMO orbital is antisymmetrical $\pi$-type orbital on the $\mathrm{C}=\mathrm{C}$ bond, presenting the greatest extension along the $\mathrm{p}_{\mathrm{z}}$ direction. According to Klopman's Charge and Frontier Orbital Control Concept ${ }^{13}$ and as shown in the figure 2 for both reactions (electrophilic addition with methylsulfide cation and nucleophilic displacement of thiiranium ion by chloride anion), the energy gap between the HOMO of the nucleophile and the LUMO of the electrophile is small compared to that between the orbitals of individual reagents, and so the reaction could be frontier-orbital controlled rather than charge-controlled. Also, as the thiiranium ion LUMO and the next lowest vacant orbital LUMO+1 present the greatest extension around the $\mathrm{C}-\mathrm{C}$ bond along the $p_{z}$ direction, they offer the greatest interaction with an occupied orbital of the nucleophile (chloride anion) when the nucleophile approaches the intermediate along this direction. Ring opening may therefore occur in the trans manner, as described in the experimental investigation. ${ }^{3,4}$ In addition, the regioselective features of thiiranium ring-opening reactions with the chloride anion may be governed by the small energy gap between the LUMO and LUMO+1 of the intermediates. ${ }^{14}$ The interaction between an occupied orbital of the nucleophile (chloride anion) approaching the $\mathrm{C}-\mathrm{C}$ atoms of the intermediate could in some cases be executed predominantly with the LUMO+1, and if this interaction is stronger than the LUMO interaction with the nucleophile it will control the course of the reaction. 


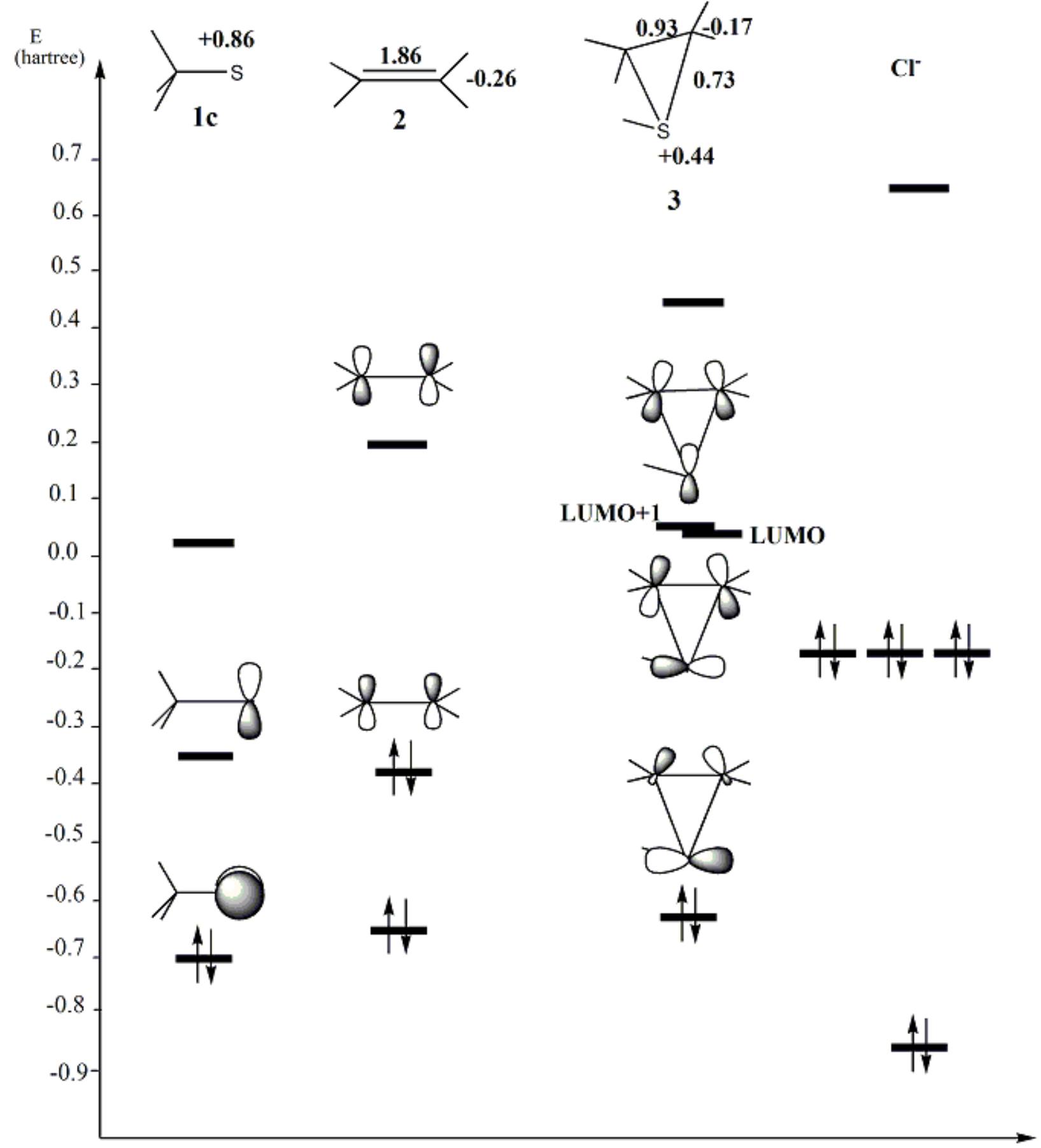

Figure 2. Frontier molecular orbital diagram with presented spatial locations (shapes), energies of HOMOs and LUMOs, also Lowdin charges, bond orders, for interacting electrophile methanesulfide cation 1c, nucleophiles - ethylene 2 and chloride anion, together with the thiiranium cation reaction intermediate 3. 
On the basis of above mentioned statements the further effort was concentrated to explain effects determining experimentally observed regioselectivity and factors controlling ring-opening reactions of differently substituted thiiranium ions.

Quantum chemical calculations were carried out to study the geometry and electronic structure of lowest energy intermediate structures for addition reaction of methylsulfide cation 1c to double bond of ethylene substituted derivatives prop-1-ene $\mathbf{2 a}$, chloroethene $\mathbf{2} \mathbf{b}$, acryloyl chloride 2c, acrylonitrilo 2d, vinylethene $2 \mathbf{e}$.

The geometry optimizations revealed few minimum energy structures from which the threemembered cyclic thiiranium cation type intermediate structures 3a-d are presented in figure 3 as most stable and lowest in energy for the addition reaction of interest.

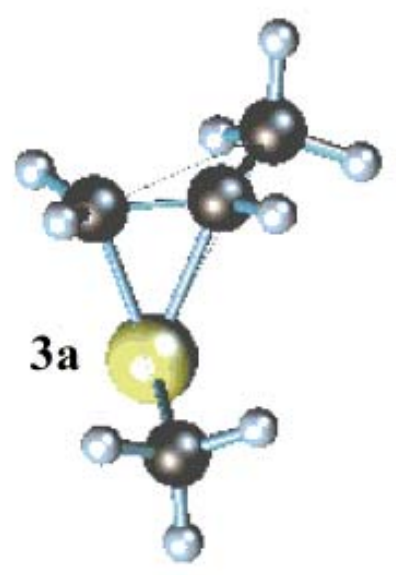

$-553.98$

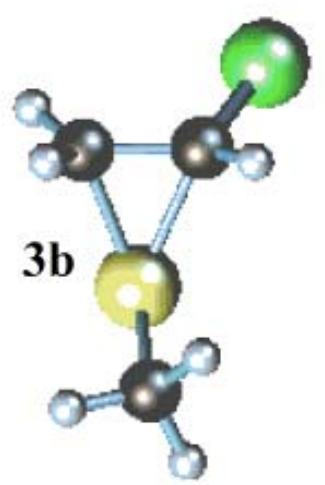

$-973.82$

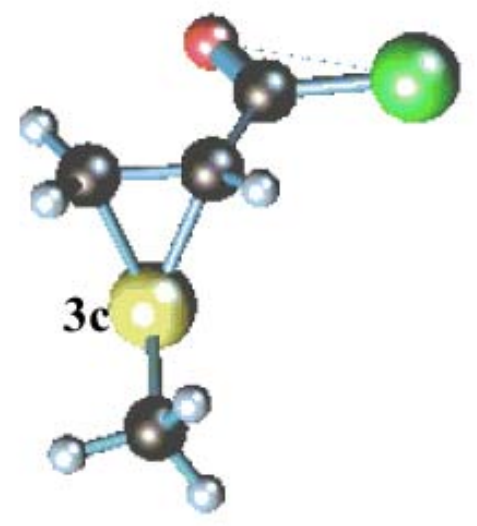

$-1086.53$

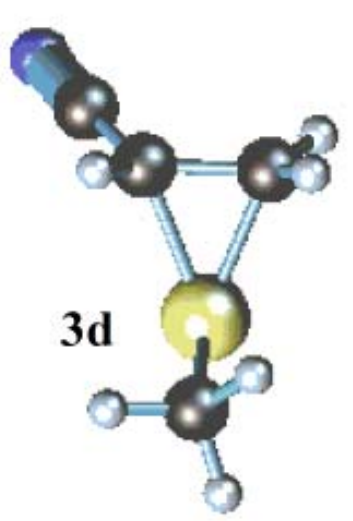

$-606.63$

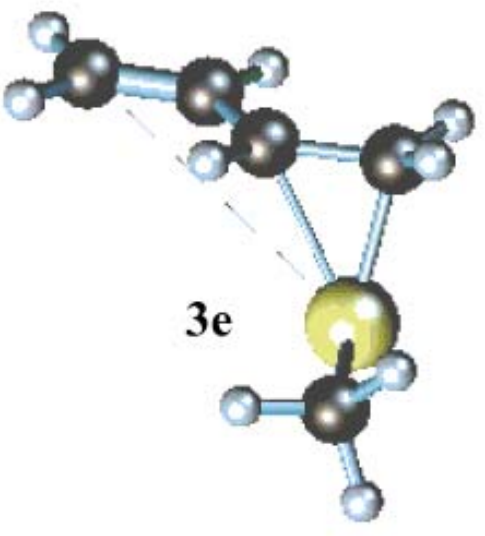

$-591.82$

Figure 3. Equilibrium geometries of intermediate structures 3a-d optimized for addition reaction of methylsulfenchloride to substituted ethenes and presented with total energies in Hartree. 
The dihedral angles $C(\alpha)-C(\beta)-S-C$ and $C(\beta)-C(\alpha)-S-C$ of thiiranium intermediate structures 3 type mostly reflect the hybridization of the $\mathrm{S}$ atom in the thiiranium fragment and should be $90^{\circ}$ for $\mathrm{p}, 180^{\circ}$ for $\mathrm{sp}^{2}$ and $125.5^{\circ}$ for $\mathrm{sp}^{3}$ hybridizations, respectively. Usually it is considered that in compounds such ethylene oxide or sulfide the lone pair of electrons of hetero atoms takes $\mathrm{sp}^{3}$ type configuration. By analogy the $\mathrm{sp}^{3}$ configuration may also be seen to be plausible for thiiranium ion intermediates, which have similar structures. In the fully optimized structures of ethylene-based thiiranium ion intermediate the dihedral angles are about $96^{\circ}$. It follows that the $\mathrm{S}$ atom loses $\mathrm{sp}^{3}$ character and more closely relates to $\mathrm{p}$ hybridization.

To develop further a qualitative understanding of the regiochemistry of thiiranium ring opening by nucleophilic displacement with chloride anion it was of the interest to examine different reactivity of the $C(\alpha)-C(\beta)$ carbon atoms of substituted ions 3a-d, estimating reactivity indexes such as the local atomic charges and reactivity indexes derived from the characteristic of the LUMOs. ${ }^{15}$

The effects of methyl, chloro, carbonyl chloride and vinyl substituents of $\alpha$-carbon on the $\mathrm{C}(\alpha)-\mathrm{C}(\beta)$ bond of the thiiranium ion 3a-d was considered performing the examination of Lowdin charges together with the examination of electrophilic Fukui functions $f^{+}(\mathrm{r}){ }^{15}$ for $\mathrm{C}(\alpha)$ and $\mathrm{C}(\beta)$ atoms involved in two vacant orbitals LUMO and LUMO+1.

Table 1. The LUMO and LUMO +1 electrophilic Fukui functions $f^{+}(\mathrm{r})$ and Lowdin charges for $\alpha$ - and $\beta$-carbon atoms of thiiranium ion intermediates 3a-e

\begin{tabular}{ccccc}
\hline No & Atom & Charge & $\begin{array}{c}\text { LUMO } \\
f^{+}(\mathrm{r})\end{array}$ & $\begin{array}{c}\text { LUMO+1 } \\
f^{+}(\mathrm{r})\end{array}$ \\
\hline \multirow{2}{*}{ 3a } & $\mathbf{C}(\alpha)$ & +0.004 & 0.408 & 0.011 \\
& $\mathbf{C}(\beta)$ & -0.107 & 0.008 & 0.289 \\
$3 \mathrm{~b}$ & $\mathbf{C}(\alpha)$ & -0.139 & 0.042 & 0.024 \\
& $\mathbf{C}(\beta)$ & -0.095 & 0.184 & 0.271 \\
$3 \mathrm{c}$ & $\mathbf{C}(\alpha)$ & -0.142 & 0.002 & 0.574 \\
\multirow{2}{*}{ 3d } & $\mathbf{C}(\beta)$ & -0.066 & 1.061 & 0.062 \\
& $\mathbf{C}(\alpha)$ & -0.281 & 0.012 & 0.255 \\
$3 \mathrm{e}$ & $\mathbf{C}(\beta)$ & -0.403 & 0.450 & 0.006 \\
& $\mathbf{C}(\alpha)$ & -0.136 & 0.2738 & 0.499 \\
\hline
\end{tabular}

The calculated Lowdin charges of $\alpha$ and $\beta$ carbon atoms for methyl, nitrilo and vinyl substituted thiiranium ions 3a, 3d and 3e show that the $\beta$ carbon is more negatively charged than $\alpha$-carbon, suggesting that the position of the $\alpha$ carbon is more favourable for nucleophilic attack by chloride anions, while for chloro and carbonyl substituted 3b-c $\alpha$-carbon is more negatively charged, suggesting that the position of the $\beta$ carbon is more favourable for nucleophilic attack by chloride anions. However, experimental results ${ }^{3-5}$ revealed the opposite situation: when $\alpha$ carbon is substituted with methyl a ring-opening reaction occurs at the $\beta$ carbon by chloride 
attack at the terminal $\beta$ carbon, giving the kinetically controlled aM adduct, in case substitution of $\alpha$-carbon with carbonyl group 3c by chlorine attack gives the $\mathbf{M}$ orientation, leading to ring opening reaction at the $\alpha$ carbon. This implies that thiiranium ring-opening regioselectivity can not be fully explained by attractive electrostatic interactions between the reacting centers.

This is in agreement with the results outlined in the preceding paragraph, which are consistent with the reaction being frontier-orbital controlled.

We therefore concentrated efforts on the examination on $f^{+}(\mathrm{r})$ and orbital locations for the two LUMOs of 3a-d, which are important for interpretation of nucleophilic displacements in thiiranium ions. In the table 1 have been presented $f^{+}(\mathrm{r})$ on $\mathrm{C}(\alpha)$ and $\mathrm{C}(\beta)$ atoms for the two lowest vacant orbitals LUMO and LUMO+1. Those orbitals are formed from out-of-plane $p_{z}$ orbitals, which result in $\pi$-complex character for all intermediates 3a-d. Replacement with methyl, chloro, carbonyl, nitrilo and vinyl substituents on the $\alpha$-carbon of the $\mathrm{C}$-C bond resulted in locations distortions of vacant orbitals for all substituted intermediates 3a-d, comparing to ethylene-based thiiranium 3. The spatial distributions of those orbitals presented in the Tables S3 and S4 of supplementary information section in this paper.

The thiiranium ion ring-opening regioselectivity is governed by whether the chloride ion attacks $C(\alpha)$ or $C(\beta)$ of the intermediate, and so is considered to be determined by unsymmetrical location extension of the vacant orbital and $f^{+}(\mathrm{r})$ on $\mathrm{C}(\alpha)$ or $\mathrm{C}(\beta)$; the larger the $f$ ${ }^{+}(\mathrm{r})$, the easier the chloride anion attack at that site. Also, as the energy differences between the LUMO and LUMO+1 are very small as shown the figure 2, it implies that nucleophilic attack of chloride could be determined by interactions depending on the greater impact of LUMO (energy gap controlled) or greater impact of LUMO+1 (orbital overlap control). ${ }^{14}$ In the case of methyl and chloro-substituted intermediates $\mathbf{3 a}, \mathbf{3 b}$ it is easy to see (table 1$)$ that the $f^{+}(\mathrm{r})$ of LUMO+1 has greater value on terminal $C(\beta)$ than on $C(\alpha)$ atom, and accordingly to the experiments ${ }^{3-5}$ it shows that the chloride anion may more easily attack the terminal $\mathrm{C}(\beta)$ to give the aM structures. In case of methyl substituted 3a aM final orientation is kinetically controlled reaction adduct, while $\mathbf{3 b}$ chloro substituted $\mathbf{a M}$ product are stable. On the other hand, in the case of carbonyl, nitrilo and vinyl substituted thiiranium ion intermediates $3 \mathbf{c}$ and 3d,e, the $f^{+}$(r) of LUMO+1 has greater value on $\mathrm{C}(\alpha)$ atom. This leads to the opposite conclusion to that for the methyl substitution case, i.e. carbonyl and vinyl and nitrilo substituted intermediates $3 \mathbf{c}$ and 3d,e gave adducts with $\mathbf{M}$ orientation governed by the initial addition of chloride to the $\alpha$-carbon atom, while the carbonyl and nitrilo substituted $\mathbf{M}$ adduct are kinetically controlled and undergoes further rearrangement to stable $\mathbf{a M}$ product. ${ }^{3-5}$ The electron populations and $f^{+}(\mathrm{r})$ of the LUMO+1 for 3a-d is consistent with the experimental possibility of forming kinetically controlled adducts. ${ }^{3-5}$

Further investigations shows that the orbital locations and $f^{+}(\mathrm{r})$ of the LUMO for thiiranium intermediates 3a-d are consistent with the possibility of rearrangement to the thermodynamically stable products. The value of the LUMOs $f^{+}(\mathrm{r})$ on $\alpha$ carbon (table 1), compared to value on $\mathrm{C}(\beta)$ in the vinyl-substituted thiiranium 3d, implies that the $C(\alpha)$ position is more susceptible to nucleophilic addition of a chlorine atom. This result is in agreement with experimental ${ }^{3-5}$ 
observations showing exceptional formation of $\mathbf{M}$-oriented products, which are thermodynamically stable and did not show any tendency to rearrange.

The LUMO electron populations of carbonyl and nitrilo substituted thiiranium 3c,d located on $\beta$-carbon. This result corresponds to experimental finding ${ }^{3-5}$ that nucleophilic displacement process of carbonyl and nitrilo substituted $\mathbf{M}$ oriented kinetically controlled adduct $\mathbf{3 c}$ undergoes rearrangement to aM products which are thermodynamically stable. In the case of nucleophilic addition of chloride to the methyl-substituted thiiranium ion 3a subsequent rearrangement of kinetically controlled $\mathbf{a M}$ adducts to stable $\mathbf{M}$ products was observed experimentally. ${ }^{3-5}$ Accordingly the calculated $f^{+}(\mathrm{r})$ for LUMO and orbital shapes for 3a shows enlarged electron population probability along the $p_{z}$ direction of the $\alpha$-carbon atom, which supports attraction to form M-oriented products.

The formation of kinetically controlled adducts is favoured by the possibility of interaction of the thiiranium ion $\mathrm{C}-\mathrm{C}$ bond with chloride, between the HOMO of nucleophile and the LUMO+1 vacant orbital of electrophile. While thermodynamically stable products formation is favoured the interaction of the HOMO of nucleophile (chloride anion) with LUMO of electrophile (thiiranium ion C-C bond).

\section{Conclusions}

The quantum chemical optimizations of the most likely intermediates indicate that the symmetrical three-cyclic thiiranium ion is a most stable intermediate structure for reactions of methylsulfen chloride with ethylene, prop-1-ene, chloroethene, vinylethene, acrylic acid chloride. For the intermediate ions in this study, the role of sulfur appears to be that of directing the stereochemistry of the addition reaction of chloride, in this case forming trans products upon nucleophilic attack on the $\mathrm{C}$-C bond of the thiiranium ion. This is in agreement with experimental results. The comparison of calculated results in vacuum and in solvated media shows that ionization of the $\mathrm{MeSCl}$ molecule by polar solvents could affect electrophilic addition reaction. The investigation of the addition of the methylsulfide cation to ethylene with subsequent attack of chloride anion shows the reaction to be frontier-orbital controlled. The regioselectivity features of thiiranium ion opening by chloride anion depend on the LUMO and LUMO+1 of thiiranium ion and the HOMO of the approaching chloride. The LUMO+1 of thiiranium ions have greatest interaction with the nucleophile approaching along $p_{z}$ direction, leading to kinetically controlled adduct formation, while the thiiranium ion LUMOs have greater interaction - leading to subsequent thermodynamically stable product formation. 


\section{Supplementary Information Available}

Table S1. Lowdin charge densities and total energies (E) in Hartrees of starting ethane 2 and substituted ethenes 2a-e calculated in vacuum and solvent model using Ab initio Hartree-Fock method with 6-311+G basis.

Table S2. Lowdin charge densities and total energies (E) in Hartrees of episulfonium intermediates 3, 3a-e calculated in vacuum and solvent model using $\mathrm{Ab}$ initio Hartree-Fock mrthod with $6-311+\mathrm{G}$ basis.

Table S3. The HOMO and LUMO spatial distribution and energies (in Hartrees) presented for starting derivatives 2, 2a-e optimized at Ab initio Hartree-Fock 6-311+G level in vacuum and in solvated media using the polarizable continuum model (PCM) approach.

Table S4. The HOMO, LUMO and LUMO+1 energies (in Hartrees) and shapes presented for reaction intermediates episulfonium derivatives 3, 3a-e optimized at Ab initio Hartree-Fock 6$311+\mathrm{G}$ level in vacuum and in solvated media using the PCM approach.

\section{Acknowledgements}

This work was supported by a grant from the Lithuanian Science Foundation (No. V-22, Reg. No. V-05016).

\section{References}

1. (a) Helten, H.; Schirmeister, T.; Engels, B. J.Org.Chem., 2005, 70, 233 (and citation herein). (b) Peterson, L.; Harris T. M.; Guengerich, F. P. J. Am. Chem. Soc., 1988, 110, 3284. (c) Gruttadauria, M.; Noto, R. J. Heterocyclic Chem., 2001, 38, 765.

2. (a) Pratt, W. B.; Ruddon, R. W.; Ensminger, W. D.; Maybaum J. Oxford University Press, Anticancer Drugs, $2^{\text {nd }}$ Edition, 1994. (b) Zhang, H.; Breido, L. Bioorg. Med. Chem. Lett., 2001, 11, 1511. (c) Klaveness, J.; Rongved, P. USA Patent Application No 20020102217, 2002. (d) Pool-Zobel, B. L.; Leucht, U. Mutat. Res., 1997, 375(2), 105. (e) Papirmeister, Br.; Gross, C. L.; Meier, H. L.; Petrali, J. P.; Johnson, J. B. Fundam. Appl. Toxicol, 1985, 5, 134.

3. (a) Rasteikiene, L.; Greiciute, D.; Linkova, M. G.; Knunyants, I. L. Rus. Chem. Rev. 1977, 46(6), 548. (b) Rasteikiene, L.; Vektariene, A.; Pociute, N.; Valaviciene, J. Chemija 1993, 1, 52. (c) Smoliakova, I. Current Org. Chem., 2000, 4(6), 589. (d) Abu-Yousef, I. A.; Hynes, R. C.; Harp, D. N. Tetrahedron Lett., 1993, 34, 4289., (e) Jones, D. K.; Liotta, D. C. Tetrahedron Lett., 1993, 34, 7209. (f) Baldwin, I; Briner, P. Org. Lett., 2002, 4, 4381. (g) Fox, D.; House, D. Angew. Chem., 2002, 114, 2572. (h) Johnston, B.; Pinto, M. J. Org. Chem., 2000, 65, 4607. 
4. (a) Mueller, W. H.; Butler, P. E. J. Org. Chem. 1968, 33, 2642. (b) Warren, A. T. J. Org. Chem. 1969, 34, 871.

5. (a) Mueller, W. H.; Butler, P. E. J. Am. Chem. Soc 1968, 90, 2075., (b); Liu, Ch.; Hashimoto, Y. Bull. Chem. Soc. Jpn. 1996, 69, 2095. (c) Bach, R. D.; Henneike, D. R. J. Am. Chem. Soc. 1970, 92, 5589.

6. (a) Johnson, C. R.; Rigau, J. J. J. Am. Chem. Soc., 1969, 91, 5398. (b) Trost, B. M. J. Am. Chem. Soc., 1973, 95, 5288.

7. Mueller, W. H. Angew. Chem. Internat. Edit. 1969, 8, 482.

8. (a) Kalaiselvan, A.; Venuvanalingam, P. TEOCHEM 2006, 763, 1. (b) Helten, H.; Schirmeister,T.; Engels, B. J.Org.Chem. 2005, 70, 233. (c) Banks, H. D.; White, W. E. ARKIVOC 2000, 1(ii), 141.

9. Dudley, T.; Smoliakova, I.; Hoffmann, M. J. Org. Chem. 1999, 64, 1247.

10. Schmidt, M. W.; Baldridge, K. K.; Boatz, J. A.; Elbert, S. T.; Gordon, M. S.; Jensen, J. H.; Koseki, S.; Matsunaga, N.; Nguyen, K. A.; Su, S. J.; Windus, T. L.; Dupuis, M.; Montgomery, J. A. J. Comput. Chem. 1993, 14, 1347.

11. (a) MOLEKEL 4.0, P. Flükiger, H.P. Lüthi, S. Portmann, J. Weber, Swiss Center for Scientific Computing, Manno (Switzerland), 2000. (b) ViewMol3D is a 3D OpenGL viewer for molecular structures, created by Andrew Ryzhkov and Arcady Antipin for visualization of molecules models. http://members.tripod.com/ RedAndr/vm3/.

12. Walsh, D. Nature 1947, 159, 165, 712.

13. Klopman, G. J. Am. Chem. Soc. 1968, 90, 223.

14. (a) Modena, G.; Pasquato, L.; Lucchini, V.; Chem. Eur. J. 2000, 6(4), 589. (b) Lucchini, V.; Modena, G.; Pasquato, L. J. Am.Chem.Soc. 1995, 117, 2297.

15. (a) Senet, P. J. Chem. Phys., 1997, 107, 2516. (b) Karelson, M.; Lobanov, V. S. Chem. Rev., 1996, 96, 1027. (c) Lepadatu, C.; Nitulescu, E. Acta Chim. Slov. 50, 539, (2003). 